\title{
HIGHLIGHTS
}

IMAGING

\section{Confocal endomicroscopy enables deeper in vivo imaging of human liver}

Scientists have successfully used confocal laser endomicroscopy (CLE) to visualize the human liver in vivo at greater imaging depths and at higher resolutions than was previously possible. The study's lead author Martin Goetz points out that the technique is safe and provides immediate histological results during laparoscopic examinations in patients with suspected liver disease.

Histopathological analysis of liver biopsy samples is regarded as the 'gold standard' in the diagnosis of liver disease, but the process confers a risk of bleeding complications and sampling error. Goetz and colleagues wanted "to monitor dynamic biological processes in humans in vivo" and had already demonstrated in an earlier study that CLE (using blue laser light and fluorescein) could be used to visualize the human liver during laparoscopy.

In the new study, Goetz et al. used a near-infrared light probe and an indocyanine green contrast for CLE of livers from 22 patients with suspected liver disease (including fatty liver disease and hepatitis). The researchers could observe subcellular liver architecture (such as fibrous tissue, bile ducts and sinusoids) in both healthy and diseased livers at imaging depths of $>350 \mu \mathrm{m}$ and at high resolution. Moreover, findings from CLE compared favorably with histological results from biopsy samples and could correctly predict the presence of steatosis and fibrosis in $81 \%$ and $90 \%$ of cases, respectively.

Goetz believes that deep tissue imaging could prove useful before endoscopic resection and permit submucosal imaging of the gastrointestinal tract.

\section{Katrina Ray}

Original article Goetz, M. et al. Near-infrared confocal imaging during mini-laparoscopy: a novel rigid microscope with increased imaging plane depth. J. Hepatol. 53, 84-90 (2010) 